\title{
Application of Fuzzy Self-Optimizing Control Based on Differential Evolution Algorithm for the Ratio of Wind to Coal Adjustment of Boiler in the Thermal Power Plant
}

\author{
Ting Hou \\ College of Electronic and Electrical \\ Engineering \\ Shanghai University of Engineering \\ Science \\ Songjiang District, Shanghai \\ 201620, China
}

\author{
Liping Zhang* \\ College of Electronic and Electrical \\ Engineering \\ Shanghai University of Engineering \\ Science \\ Songjiang District, Shanghai \\ 201620, China
}

\author{
Yuchen Chen \\ College of Electronic and Electrical \\ Engineering \\ Shanghai University of Engineering \\ Science \\ Songjiang District, Shanghai \\ 201620, China
}

\begin{abstract}
The types of coal are multiplex in domestic small and medium sized boilers, and with unstable ingredients, the method of maintaining the amount of wind and coal supply in a fixed proportion of the wind adjustment does not always ensure the best economical boiler combustion process, the key of optimizing combustion is to modify reasonable proportion of wind and coal online. In this paper, a kind of fuzzy selfoptimizing control based on differential evolution algorithm is proposed, which applied in the power plant boiler system, the boiler combustion efficiency has been significantly improved than previous indirect control. In this paper, a thermal power plant is our research object, in the case of determining the optimum system performance, the unit efficiency can be increased significantly using this method, and the important issues of energy efficiency of power plants can be successfully solved.
\end{abstract}

Keywords-fuzzy self-optimizing control; differential evolution algorithm; best ratio of wind to coal; boiler efficiency

\section{INTRODUCTION}

Boiler combustion control system is a nonlinear system, and with the characteristics of time-varying, noise disturbing, pure hysteresis, the experience of the operator can't be used with the conventional control methods. But in the process of the actual operation of the boiler, these experiences information is important. Optimal control of modern control theory is also difficult to be applied in the modelling of such systems. However, fuzzy control can be just applied to control the unknown or changing process for this kind of mathematical model [1].Using fuzzy theory and fuzzy control method, the experience of the operator can be summed up. Moreover, a kind of fuzzy control strategy can be achieved using this fuzzy logic inference method.

In this paper, thermal power plant is our research object, the power plant is small, and it has three $12 \mathrm{MW}$ units, 3 sets of $150 \mathrm{t} / \mathrm{h}$ coal-fired boiler. The self-optimizing simulation is used to find the best ratio of wind to coal, and combining differential evolution algorithm, the quantization factor $\mathrm{K}_{\mathrm{y}}$ will be optimized, then fuzzy control technology is used to regulate the speed of blower speed and coal feeder, finally 3 boilers of the thermal power plant cogeneration combustion efficiency significantly will be improved than previous indirect control, which is agreed with our above analysis.

\section{BOILER FUZZY SELF-OPTIMIZING CONTROL SYSTEM AND DIFFERENTIAL EVOLUTION ALGORITHM}

\section{A. PID fuzzy controller system}

The error and error change rate between the amount transferred and the fixed value as input variables are always used in the general fuzzy controller; therefore, it has the similar effect with the conventional PD controller ${ }^{[2]}$. A good dynamic quality can be obtained using such fuzzy controller in control system. However, steady-state error of the amount transferred is difficult to eliminate. In order to eliminate the steady-state error of the control system, PID fuzzy control system can be used as shown in Fig1. In the figure, e and $\dot{e}$ are respectively the error and error derivative. Conventional control table form is used for fuzzy control.

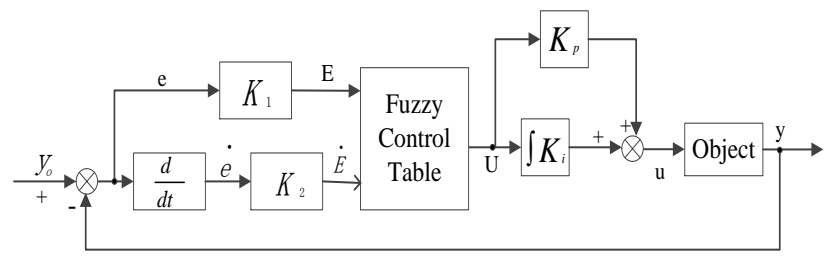

Fig. 1. PID fuzzy controller schematics

Output control table need to be changed to the amount of actual control value through an output link, and then coupled to the controlled object to achieve the control. Commonly, the two ways of output link used are proportional output and integral output, the former has a fast step response characteristic, but it is error control; the latter can be close to the no error control, but with the slow response and the 
overshoot is relatively large. The combination of both ways is used in the system, which have advantages of a small overshoot and short time transient.

Mathematical expression of PD-type fuzzy controller can be derived by algebraic product-addition-focus fuzzy inference method:

$$
\mathrm{u}=\mathrm{A}+\mathrm{Pe}+\mathrm{De}
$$

$$
\text { In formula (1), } \begin{aligned}
A & =u_{i, j}-\frac{u_{i+1, j}-u_{i, j}}{e_{i+1}-e_{i}} e_{i}-\frac{u_{i+1, j}-u_{i, j}}{\dot{e}_{j+1}-\dot{e}_{j}} \dot{e}_{j} \\
D & =\frac{u_{i, j+1}-u_{i, j}}{\dot{e}_{j+1}-\dot{e}_{j}} \\
P & =\frac{u_{i+1, j}-u_{i, j}}{e_{i+1}-e_{i}}
\end{aligned}
$$

Thus input-output relationship of the input and output PID fuzzy controller can obtained as shown in Figure 1:

$$
\begin{aligned}
\mathrm{u}= & \mathrm{K}_{\mathrm{p}} \mathrm{U}+\mathrm{K}_{\mathrm{i}} \int \mathrm{Udt}= \\
= & \mathrm{K}_{\mathrm{p}}\left(\mathrm{A}+\mathrm{PK}_{1} \mathrm{e}+\mathrm{DK}_{2} \dot{\mathrm{e}}\right)+ \\
& \mathrm{K}_{\mathrm{i}} \int\left(\mathrm{A}+\mathrm{PK}_{1} \mathrm{e}+\mathrm{DK}_{2} \dot{\mathrm{e}}\right) \mathrm{dt} \\
= & \mathrm{K}_{\mathrm{p}} \mathrm{A}+\mathrm{K}_{\mathrm{i}} \mathrm{At}+\left(\mathrm{K}_{\mathrm{p}} \mathrm{K}_{1} \mathrm{P}+\mathrm{K}_{\mathrm{i}} \mathrm{K}_{2} \mathrm{D}\right) \\
+ & \mathrm{K}_{\mathrm{i}} \mathrm{K}_{1} \mathrm{P} \int \mathrm{edt}+\mathrm{K}_{\mathrm{p}} \mathrm{K}_{2} \mathrm{De}
\end{aligned}
$$

The above mentioned fuzzy controller have four adjustable parameters: quantify factors $\mathrm{K}_{1} 、 \mathrm{~K}_{2}$, scale factor $\mathrm{K}_{\mathrm{p}}$ and integral coefficient $\mathrm{Ki}$, increasing $\mathrm{K}_{1} 、 \mathrm{~K}_{2}$, the resolution of the error can be improved, then the control accuracy is also improved. But if $K_{1} 、 K_{2}$ are too big, it will bring unstable for the system ${ }^{[3]}$. Increasing $\mathrm{K}_{\mathrm{p}}$ or $\mathrm{Ki}$, response speed will increase, but which will lead to oscillate. Based on actual adjusted experience, the desirable value as $\mathrm{K}_{1} \approx \mathrm{K}_{2}, \mathrm{~K}_{\mathrm{p}}=(2 \sim 3) \mathrm{K}_{\mathrm{i}}$. When $\mathrm{K}_{1}$ and $\mathrm{K}_{2}$ are too larger, $\mathrm{K}_{\mathrm{p}}$ and $\mathrm{K}_{\mathrm{i}}$ should be decreased, and when the sampling period is longer, then $K_{p}$ and $K_{i}$ can be chosen larger.

\section{B. Fuzzy self-optimizing controller in the ratio of wind to coal}

In the boiler control, due to the amount of pulverized coal cannot be accurately measured online, so the excess air coefficient $\alpha$ is used to substitute $\beta$ which represents the ratio of wind to coal volume, so ratio of wind to coal and $\alpha$ are equivalent. The ratio of actual air volume (V) and the theoretical air amount $\left(\mathrm{V}_{\mathrm{o}}\right)$ is the excess air factor $\alpha$. According to "Safety Engineering Dictionary", exhaust of the boiler is qualified when the excess air coefficient $\alpha$ is within the range of 1.15 to 1.25 of the thermal power plant ${ }^{[4]}$, in addition, the thermal efficiency is highest at this time. If excess air coefficient $\alpha$ is too large, excess air flue gas will take too much heat, increasing exhaust gas temperature will lead to heat loss $\mathrm{q}_{2}$ increasing, at the same time, it will produce a large amount of $\mathrm{NO}_{\mathrm{x}}$ and $\mathrm{SO}_{\mathrm{x}}$ pollution; on the contrary, if $\alpha$ is too small, complete combustion of fuel cannot be guaranteed. Selecting the appropriate $\alpha$ is an important means to reduce boiler heat loss and improve thermal efficiency.

In this system, $\alpha$ is the seeking optimization index, if:

$$
\alpha \notin[1.15,1.25]
$$

Start fuzzy self-optimizing search, when $\alpha$ is within the range of 1.15 to 1.25 , indicates that the optimum working range has been found, and then optimization can be stopped.

Self-optimizing fuzzy controller works as follows: combustion coal as an index used to find the best ratio of wind to coal ${ }^{[5]}$ with fuzzy self-optimizing controller. Increment of coal consumption $\Delta \mathrm{y}$ will be measured at each sampling period, accord to $\Delta y$ and the optimization step of previous cycle to determinate the optimization step. $\Delta \mathrm{Y}$ and $\Delta \mathrm{X}$ are coal consumption and fuzzy linguistic variables of the steps. $\mathrm{K}_{\mathrm{y}}$ is quantization factor of $\Delta y$, which converts the fuzzy linguistic variables of coal consumption increment $\Delta \mathrm{Y}$ to the actual value, $K_{x}$ is scale factor, which convert the $\Delta X$ to actual value of the step.

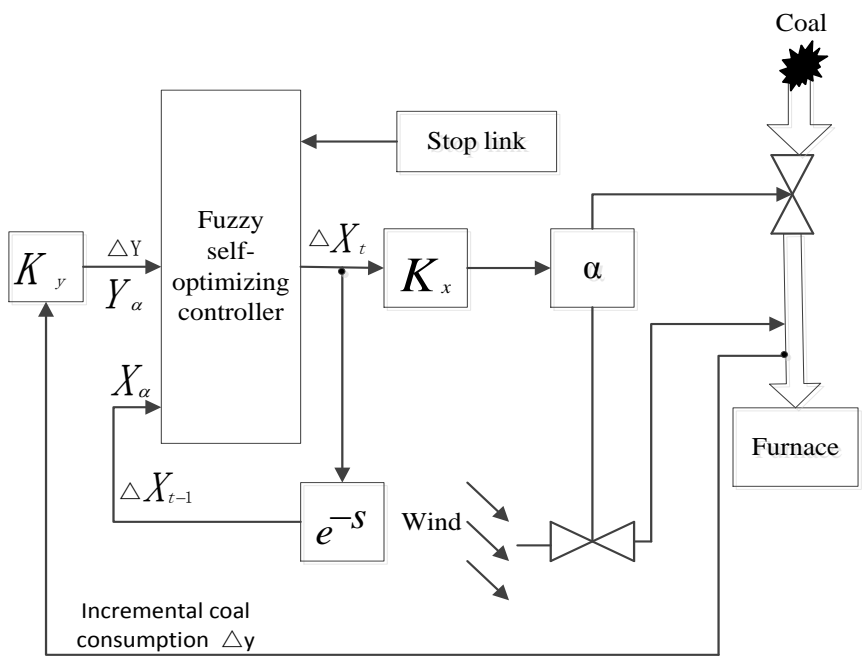

Fig. 2. Fuzzy self-optimizing controllor based on differential evolution algorithm for the ratio of wind to coal

In practical applications, in order to ensure the stability of self-optimizing process, a stop link ${ }^{[6]}$ is added. If the temperature of the furnace has a big fluctuation due to environment distribution, the search should be stopped to avoid malfunction.

Selecting $\Delta \mathrm{Y}, \Delta \mathrm{X}$ respectively as 8 and 6 linguistic variables of fuzzy subsets is contained as follows:

$$
\begin{aligned}
& \Delta \mathrm{Y}=\{\mathrm{NB}, \mathrm{NM}, \mathrm{NS}, \mathrm{NO}, \mathrm{PO}, \mathrm{PS}, \mathrm{PM}, \mathrm{PB}\} \\
& \Delta \mathrm{X}=\{\mathrm{NB}, \mathrm{NM}, \mathrm{NS}, \mathrm{PS}, \mathrm{PM}, \mathrm{PB}\}
\end{aligned}
$$

NB, NM, NS, NO, PO, PS, PM, PB respectively denote negative big, negative medium, negative small, negative zero, positive zero, positive small, positive medium and positive big.

The fuzzy domain of $\Delta \mathrm{Y}$ and $\Delta \mathrm{X}$ are defined as 14 and 12 grades: 
$\mathrm{Y}_{\alpha}=\{-6,-5,-4,-3,-2,-1,-0,+0,+1,+2,+3,+4,+5,+6\}$

$\mathrm{X}_{\alpha}=\{-6,-5,-4,-3,-2,-1,+1,+2,+3,+4,+5,+6\}$

Self-optimizing search process control rules showed as in Table I. $\Delta \mathrm{X}_{\mathrm{t}-1}$ is the optimization step of previous cycle, $\Delta \mathrm{X}_{\mathrm{t}}$ is the optimization step. Table II shows the rule table about fuzzy self-optimizing control in the ratio of wind to coal.

TABLE I. RULE TABLE ABOUt FuZZY SELF-OPTIMIZING CONTROL IN THE RATIO OF WIND TO COAL

\begin{tabular}{c|cccccc}
\hline$\Delta \mathrm{X}_{\mathrm{t}} \Delta \mathrm{X}_{\mathrm{t}-1}$ & & & & & & \\
& $\mathrm{NB}$ & $\mathrm{NM}$ & $\mathrm{NS}$ & $\mathrm{PS}$ & $\mathrm{PM}$ & $\mathrm{PB}$ \\
\hline NB & & & & & & \\
NM & PB & PB & PB & NB & NB & NB \\
NS & PS & PM & PB & NB & NB & NM \\
NO & PS & PS & PS & NS & NM & NS \\
PO & PS & PS & PS & NS & NS & NS \\
PS & NS & NM & NM & PM & PM & PS \\
PM & NM & NB & NB & PB & PB & PM \\
PB & NB & NB & NB & PB & PB & PB \\
\hline
\end{tabular}

Control strategy of the controller can be summarized as follows:

$$
\begin{aligned}
& \text { IF } \Delta \mathrm{X}_{\mathrm{t}-1}=\mathrm{NB} \text { AND } \Delta \mathrm{Y}=\mathrm{NB} \text { THEN } \Delta \mathrm{X}_{\mathrm{t}}=\mathrm{PB} \\
& \text { IF } \Delta \mathrm{X}_{\mathrm{t}-1}=\mathrm{NB} \text { AND } \Delta \mathrm{Y}=\mathrm{NM} \text { THEN } \Delta \mathrm{X}_{\mathrm{t}}=\mathrm{PM}
\end{aligned}
$$

Apply synthesis rules of fuzzy inference, and coupled with manual correction, the ultimate self-optimizing control table can be obtained as shown in Table II.

TABLE II. TABLE ABOUT FuZZY SELF-Optimizing CONTROL IN THE RATIO OF WIND TO COAL

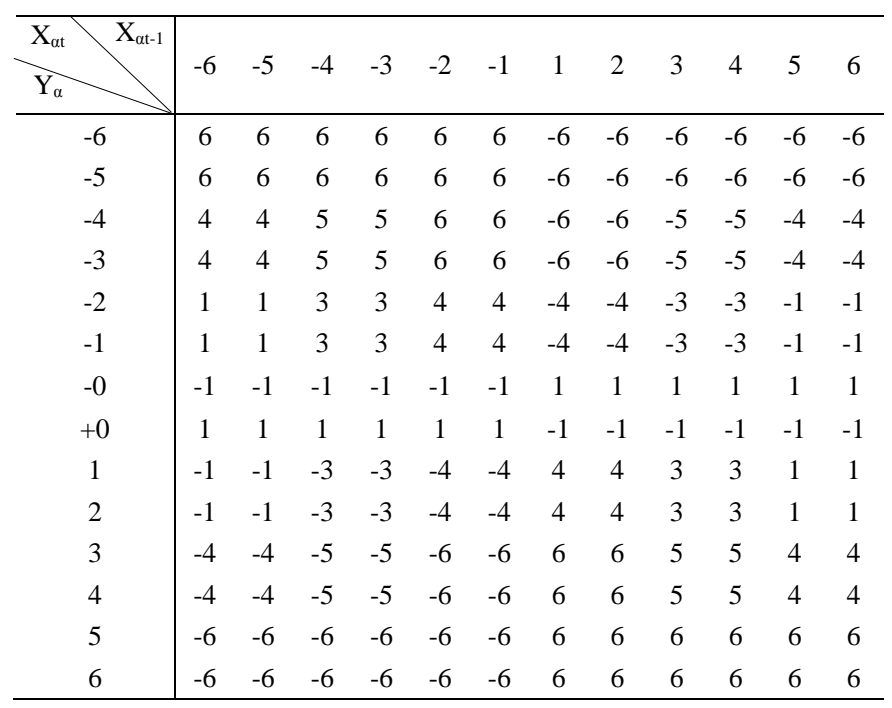

The search speed can be improved by increasing $K_{x}$ and $K_{y}$, the value of $\mathrm{K}_{\mathrm{x}}$ can also affect the loss of search, so $\mathrm{K}_{\mathrm{y}}$ can be chosen based on the search speed requirements, $\mathrm{K}_{\mathrm{x}}$ can be chosen according to the requirements of the loss of search. The differential evolution algorithm is added in the system to optimize the value of the quantization factor $\mathrm{K}_{\mathrm{y}}$, making the fuzzy value of incremental coal consumption can be conversed more precise to the actual value after each optimization, boiler efficiency ratio is improved significantly, and especially at low load.

\section{Differential evolution algorithm}

The basic idea of differential evolution algorithm is: the individuals of current population followed through mutation and crossover operation and produce tested individual, then, based on the greedy thoughts, the optimum individual is selected; thereby the new populations are generated. When in the process of $t$ times iteration, the $\mathrm{i}$-th population of individuals $x_{i}^{t}=\left(x_{i 1}^{t}, x_{i 2}^{t}, \cdots, x_{i d}^{t}\right), i=1,2, \cdots N_{p}$ is a d-dimensional candidate solution vector, the individual with best fitness value of all individuals are denoted $\mathrm{x}_{\text {best }}^{\mathrm{t}}=\left(\mathrm{x}_{\text {best }}^{\mathrm{t}}, \mathrm{x}_{\text {best }}^{\mathrm{t}}, \cdots, \mathrm{x}_{\text {best }}^{\mathrm{t}}\right)$, then three-steps operation as mutation, crossover, selection are performed in the process of the $t$-th iterations ${ }^{[7]}$.

\section{1) Mutation}

Mutation operation is achieved by DE algorithm with the differential method. There are a variety of differential strategies, common differential strategy is to select randomly two different individuals in the $t$-th generation of the population, after multiplying the vector difference of the two individual with variability factor, and a synthetic new individuals with upcoming individual variation $u_{i}^{t}, u_{i}^{t}$ is called variation vector. DE/rand/1 variation formula as follows:

$$
u_{i d}^{t}=x_{r_{1 j}}^{t}+F \cdot\left(x_{r_{2 j}}^{t}-x_{r_{3 j}}^{t}\right)(j=1,2, \cdots d)
$$

There, $u_{i j}^{t}$ is $j$-dimensional components of variation vector $u_{i}^{t}$, $\mathrm{F}$ is variability factor, used to adjust the differential scaling of multiple, usually selected as the value between $(0,1]$, three random numbers $r_{1} 、 r_{2} 、 r_{3}$ are different from each other and not equal to $\mathrm{i}, \mathrm{x}_{\mathrm{r}_{1}}^{\mathrm{t}}$ is called a parent basis vectors, $\left(\mathrm{x}_{\mathrm{r}_{2}}^{\mathrm{t}}-\mathrm{x}_{\mathrm{r}_{3}}^{\mathrm{t}}\right)$ is a parent difference vector, then crossover to $x_{i}^{t}$ and variation vector $u_{i}^{t}$ are implemented.

\section{2) Crossover}

I-th individual $x_{i}^{t}$ and variation vector $u_{i}^{t}$ generated by mutation work as the following crossover:

$$
y_{i j}^{t}=\left\{\begin{array}{l}
u_{i j}^{t}, \text { if rand } \leq C_{R} \\
x_{i j}^{t}, \text { otherwise }
\end{array}(j=1,2, \cdots, d)\right.
$$

There, $\mathrm{y}_{\mathrm{ij}}^{\mathrm{t}}$ is the individual of $\mathrm{x}_{\mathrm{i}}^{\mathrm{t}} \mathrm{j}$-dimensional components corresponding to the test individual $\mathrm{y}_{\mathrm{i}}^{\mathrm{t}}$, rand is random numbers distributed uniformly between $[0,1], \mathrm{C}_{\mathrm{R}}$ is cross-factor, usually the value is pre-set within the range $[0,1]$, cross factor determines the proportion of each component of variation vector $u_{i}^{t}$ in the tested individuals $y_{i}^{t}$. When $C_{R}=1$, tested individual is equal to the variation individual.

\section{3) Selection}

Comparing produced tested individual $y_{i}^{t}$ with the individual $\mathrm{x}_{\mathrm{i}}^{\mathrm{t}}$, the optimum individual of both individual is selected as the next generation. Therefore, comparing the fitness of tested individualy ${ }_{i}^{t}$ and individual $x_{i}^{t}$, when the fitness of tested individualy ${ }_{i}^{t}$ is better than the fitness of individuals $x_{i}^{t}$, $y_{i}^{t}$ will replace individual $x_{i}^{t}$ and become the next generation of 
individuals $x_{i}^{t+1}$, otherwise, $x_{i}^{t}$ will be retained and become individual $\mathrm{x}_{\mathrm{i}}^{\mathrm{t}+1}$ of generation $\mathrm{t}+1$.

$$
x_{i}^{t+1}=\left\{\begin{array}{l}
y_{i}^{t}, \text { if fitness }\left(y_{i}^{t}\right) \leq \operatorname{fitness}\left(x_{i}^{t}\right) \\
x_{i}^{t}, \text { otherwise }
\end{array}\right.
$$

When the fitness of $x_{i}^{t+1}$ is better than the fitness of $x_{b e s t}^{t+1}$, update $x_{\text {best }}^{t+1}$, make $x_{\text {best }}^{t+1}=x_{i}^{t+1}$, and save the best fitness

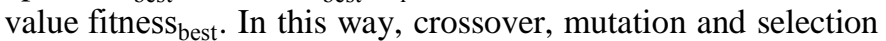
operations should be repeated, until the population optimal adaptation meet the pre-set threshold value or reach the maximum number of iterations. At this point the best fitness value corresponding to the individual is the optimal solution searched by DE algorithm for optimizing this problem. The system uses differential evolution algorithm to optimize quantitative factor $\mathrm{K}_{\mathrm{y}}$ to improve the efficiency of the boiler system, especially in low-load operation.

\section{EFFECT OF THIS SYSTEM AFTER APPLIED TO THE THERMAL POWER PLANT}

\section{A. Improvement of boiler efficiency}

Boiler efficiency is the average in a certain statistical cycle, the average efficiency of a reference period is calculated as follows:

$$
\eta=9143 \frac{\sum \mathrm{D}_{\mathrm{N}}}{\sum \mathrm{B}_{\mathrm{N}}} \%
$$

In formula (7), $\sum \mathrm{D}_{\mathrm{N}}$ represents the standard steam production during the reference period; $\sum \mathrm{B}_{\mathrm{N}}$ represents the standard coal consumption during the reference period.

Fuzzy self-optimizing controller in the ratio of wind to coal is used in unit, the coal amount is fed back to input value, change the wind volume to change the ratio of wind to coal to achieve the high efficiency of the boiler, the simulation results shown in Figure 3.

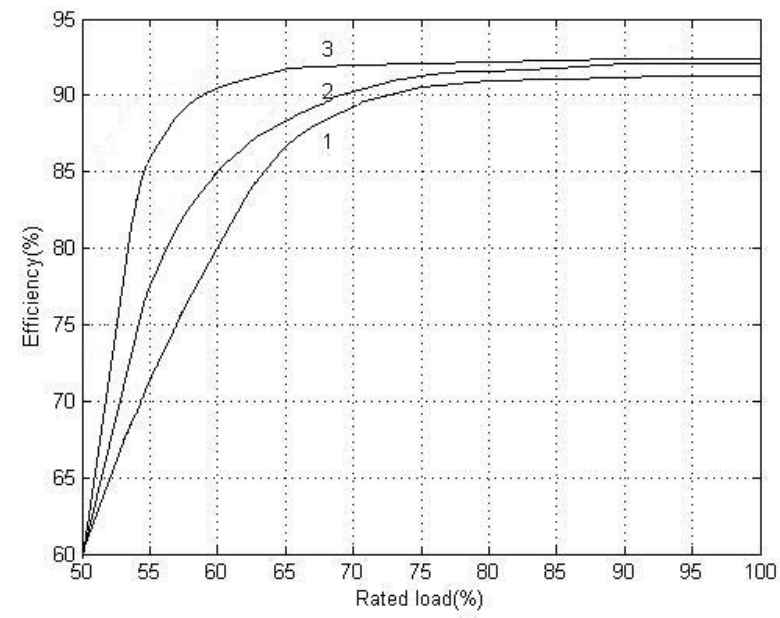

Fig. 3. Contrast curve of boiler efficiency

In figure 3 , curve 1 is the boiler efficiency when manually adjusting the ratio of the wind to coal of the system, curve 2 is boiler efficiency after adding self-optimizing fuzzy control, and curve 3 is boiler efficiency after using differential evolution algorithm to optimize quantitative factors $\mathrm{K}_{\mathrm{y}}$. As seen from figure 3 , when running with full capacity, boiler efficiency is improved as approximately $0.9 \%$ after using fuzzy selfoptimizing controller in the ratio of wind to coal than manual control, and incorporating the differential evolution algorithm into the boiler system, its efficiency can increased by approximately $0.2 \%$, especially in low-load operation, efficiency is more significant.

\section{B. Saving coal and emission reduction of the power}

Restricted by detection technology and indirect control, improper wind supply often causes a substantial loss of heat or incomplete combustion of fuel. The best control system based on the current thermal power plants can only guarantee the energy conversion efficiency is generally about $35 \%{ }^{[8]}$, it is already a very high conversion efficiency of burning calories, the average thermal efficiency of thermal power plants is about $32.5 \%$, system efficiency can be increased by $1.1 \%$ when running with full capacity, so in this way we can get considerable energy savings.

Note: The standard coal calorific value calculated using $7000 \mathrm{Kcal} / \mathrm{kg}$. The average heating ratio of thermal power plant is about $84.58 \%$.

TABLE III. ENERGY CONSUMPTION OF THE 12 MW UNIT

\begin{tabular}{cccc}
\hline & $24 \mathrm{~h}$ & $30 \mathrm{~d}$ & $365 \mathrm{~d}$ \\
\hline $\begin{array}{c}\text { Output power } \\
/ \mathrm{kWh}\end{array}$ & $0.2714 \times 10^{6}$ & $8.143 \times 10^{6}$ & $99.07 \times 10^{6}$ \\
Heat input /Kcal & $158.71 \times 10^{7}$ & $46764.69 \times 10^{7}$ & $60976.937 \times 10^{7}$ \\
$\begin{array}{c}\text { Coal } \\
\text { consumption of } \\
\text { unit/t }\end{array}$ & 226.72 & 6680.67 & 87109.97 \\
\hline
\end{tabular}

TABLE IV. ENERGY SAVINGS OF THE 12 MW UNIT

\begin{tabular}{cccc}
\hline & $24 \mathrm{~h}$ & $30 \mathrm{~d}$ & $365 \mathrm{~d}$ \\
\hline $\begin{array}{c}\text { Output power } \\
/ \mathrm{kWh}\end{array}$ & $0.2743 \times 10^{6}$ & $8.2335 \times 10^{6}$ & $100.16 \times 10^{6}$ \\
$\begin{array}{c}\text { Saving energy } \\
/ \text { Kcal }\end{array}$ & $4.45 \times 10^{7}$ & $133.04 \times 10^{7}$ & $1624.79 \times 10^{7}$ \\
$\begin{array}{c}\text { Saving standard } \\
\text { coal } \\
\text { consumption } / \mathrm{t}\end{array}$ & 2.494 & 74.789 & 957.167 \\
\hline
\end{tabular}

It can be seen from Table III and Table IV, the energysaving effect is obvious after using the control method in this article.

Combining with fuzzy self-optimizing control method based on differential evolution algorithm in this article, the boiler combustion control technology is achieved, applied in the three $12 \mathrm{MW}$ units in the thermal power plant, its efficiency is increased by $1.1 \%$, a boiler can save 957 tons of standard coal one year, so three units can save about 2,871 tons of standard coal in a year. This has a significant effect on energysaving, environmental protection, and reducing atmospheric dust pollution for the plant. 


\section{CONCLUSION}

Fuzzy self-optimizing controller in the ratio of wind to coal is used in the unit with appropriate modification, the coal amount is fed back to the forward input value, and so that ventilation can rapidly changing with the amount of fuel changes, and the ratio of wind to coal can be controlled within an appropriate range.

Differential evolution algorithm combined with fuzzy selfoptimizing control system enables the boiler operation efficiency significantly improves especially in the situation of low load.

In the case of determining the optimal performance of the system, fuzzy control technology used to make the wind volume can quickly search for the best value, improve the thermal efficiency of the boiler, achieve the effect of economizer and efficiency, and the economics of the thermal power plant is improved greatly.

\section{ACKNOWLEDGMENT}

The project has been supported by the Innovation Program of Shanghai Municipal Education Commission "Vehicle
Collision Avoidance System based on Vehicle Wireless Communication" (No.12YZ151).

\section{REFERENCES}

[1] Ji Chang-an et al. Image processing and transformation of power plant application of fuzzy control[J]. High Voltage Engineering, 2007(3311).

[2] Mao Pei.Research Minhang Power Plant 125MW unit of energy-saving technology to run[D]. Shanghai Jiaotong University,2004.

[3] Bai Ruixiang, Tai Xinmeng, Li Huien. Fuzzy Self-optimizing Control on Combustion System of Industrial Boiler [J]. Instrument Technique and Sensor, 2009, 6 (98-100).

[4] Cui Keqing.Safety Engineering Dictionary [M].Beijing: Chemical Industry Press, 1995(342-350).

[5] Ai Hong.Dynamic Fuzzy External Control of the Efficiency of Boiler Combustion[J]. Techniques of Automation \& Applications,2002,21(58).

[6] Li Shiyong. Fuzzy control [M].Harbin: Harbin institute of technology press. 2011(172-177).

[7] Wu Lianghong, Wang Yaonan, Yuan Xiaofang,Zhang Jian.Research on Differential Evolution Algorithm for MOPs[J]. Joumal of Hunan University(Natural Sciences),2009,36,2(53-57).

[8] Ling Weiping.The use of fuzzy control technology of wind and coal ratio to the design of power plant $[\mathrm{J}]$. Northeast Electric Power Technology,2011,7(43-45). 